\title{
Probabilistic vs. Sequence-Based Rendezvous in Channel-Hopping Cognitive Networks
}

\author{
Jelena Mišić, Senior Member, IEEE and Vojislav B. Mišić, Senior Member, IEEE
}

\begin{abstract}
Rendezvous in cognitive networks refers to the ability of cognitive nodes to find each other and form a network, or to find and join an already operating cognitive network. Two main approached to rendezvous have emerged: sequence-based mechanism that guarantees maximum time-to-rendezvous and blind random hopping resilient to unpredictable primary user activity. In this paper we develop analytical models for time to rendezvous in the presence of primary user activity for the orthogonal sequence-based mechanism and a blind rendezvous mechanism integrated with a transmission tax-based MAC protocol with cooperative sensing. Our analysis shows that the blind mechanism performs better under random primary user activity, the difference being more pronounced when the number of channels is high and/or primary user activity is more intense. In addition, the probabilistic mechanism allows rendezvous with either an emergent or a fully operational $\mathrm{CH}$ CPAN piconet without any interruption, unlike the sequence-based mechanism which precludes any data exchange during the rendezvous process.
\end{abstract}

Index Terms-opportunistic spectrum access; channel hopping cognitive networks; piconet formation; rendezvous protocol

\section{INTRODUCTION}

The paradigm of opportunistic (dynamic or cognitive) spectrum access (OSA) promises to improve the utilization of the available spectrum [1]. In this scheme, a number of channels is set aside for shared use by primary (i.e., licensed) and secondary, non-licensed users. However, secondary users can use the channel only in the absence of primary user activity; if a primary user appears on the working channel, secondary users should move away to another channel. This may be accomplished through dynamic channel hopping [20] in which secondary users form piconets that switch channels according to a common hopping sequence. To avoid collisions with primary users, the hopping sequence must be dynamically adapted to primary user activity. Due to their resemblance to more traditional personal area networks, we refer to such networks as channel-hopping cognitive personal area networks, or CH-CPANs.

To establish communication, a cognitive node must first undertake the so-called rendezvous procedure in which it attempts to meet another cognitive node (or an entire piconet)

- J. Mišić and V. B. Mišić are with the Department of Computer Science, Ryerson University, Toronto, ON, Canada M5B $2 K 3$.

E-mail:jmisic@scs.ryerson.ca,vmisic@ryerson.ca at the same RF channel and exchange synchronization data [12], [15]. The problem is particularly challenging in cognitive networks that rely on adaptive channel hopping [20], because the individual hopping sequences are not only different, but may change in time as well.

A number of approaches to the rendezvous problem have been proposed, with or without the aid of a dedicated infrastructure such as a central (base) station or a common control channel [11]. For obvious reasons, the latter approaches, collectively referred to as blind rendezvous [4], are preferable in practice.

A number of blind rendezvous mechanisms have focused on scenarios in which two nodes hop through the channel set according to a predefined sequence until they meet and establish communication [5], [12], [14], [22], [24]. Through careful construction of the channel hopping sequence, a finite upper bound for TTR may be guaranteed, but only in the absence of primary user activity (a collision with primary user transmission may destroy a rendezvous and extend the TTR beyond its theoretical upper bound). In fact, we show that random primary user activity transforms the TTR of sequencebased rendezvous algorithms into a random variable without a finite upper bound, thus eliminating the main advantage of such algorithms. We have used the orthogonal sequence technique from [12] as a representative sequence-based rendezvous protocol, but other such protocols would be affected by primary user activity in the same manner.

A smaller group of rendezvous mechanisms utilize pseudorandom channel hopping, either in entirely blind fashion, or aided by the knowledge about primary user activity patterns. These mechanisms can't guarantee a finite upper bound for time-to-rendezvous (TTR) on account of their probabilistic nature; but that same nature makes the collisions with primary user transmissions less damaging than in the case of sequence-based mechanisms. We analyze the performance of a recently proposed rendezvous mechanism [33] integrated in the transmission tax-based MAC protocol [34] with cooperative spectrum sensing [28]. The combined protocol not only achieves better performance than the orthogonal sequencebased mechanism under certain conditions, but also allows rendezvous to be achieved during piconet formation as well as during normal operation.

The rest of the paper is structured as follows: Section 2 surveys related work and highlights the problems of rendezvous in 
cognitive PANs in more detail. Section 3 describes the blind rendezvous mechanism in the transmission tax-based MAC protocol, while Section 4 presents the orthogonal sequencebased mechanism. The transmission tax-based MAC protocol itself, as well as the analytical models for the probabilistic and sequence-based rendezvous protocols, are described in detail in the Online Supplement. Section 5 compares the performance results obtained by solving the analytical models. Finally, Section 6 concludes the paper and highlights some future research.

\section{Related WORK}

In recent years a number of MAC protocols have been proposed for cognitive ad hoc and personal area networks [10]. Many protocols rely on the presence of a common control channel (CCC), which is assumed to be free from interference from primary users and other cognitive ad hoc networks in the vicinity [9], [26]. A number of algorithms for selection and maintaining of CCC have been proposed, including both static (dedicated) [2] or dynamic CCC, the latter being achieved through a variant of CSMA [8] or using a rendezvous-based technique when channel hopping is deployed [6], [25].

Integration of sensing, reporting and data phases in cognitive networks has been considered in [2] where nodes deploy dynamic ID numbers in order to regulate access to the medium. A two-level MAC for opportunistic spectrum access which deploys slotted ALOHA and CSMA for node access was proposed in [8] while integration of spectrum sensing rules with CSMA/CA was considered in [19]. We also note the proposal in [37] which attempts to integrate cognitive MAC functions with the power saving mode of IEEE 802.11 DCF. Recently, a MAC protocol has been proposed that integrates data transmission/reception with sensing activities that aim to ensure smooth operation under unpredictable primary user activity [34].

Regarding the rendezvous problem, a recent survey of results obtained so far can be found in [36]. Some solutions rely on the services of a central controller (e.g., [7]) or the availability of a dedicated common control channel [6], [9], [11], [26]. While both approaches promise good performance, i.e., short mean value and small (and provable) upper bound for TTR, their prerequisites are difficult to achieve and maintain in practice. Consequently, a blind rendezvous protocol appears to be much better suited to truly distributed and autonomous operation of cognitive networks [4], [36].

Two main categories of blind rendezvous protocols have emerged over time. Protocols from one group are based on predefined deterministic channel hopping sequences [5], [12], [13], [14], [22], [24], [36], [38]. In this case, the channel hopping sequence may be constructed in such a way that a finite upper bound for TTR is guaranteed, usually for two nodes finding each other and establishing communication but sometimes for larger groups as well. The other group of rendezvous protocols relies on spectrum sensing and probabilistic channel selection, possibly aided by knowledge about primary user activity patterns obtained through some kind of learning [11], [15], [18], [33].
While the existence of a finite upper bound appears to give a definite advantage to the former group, many of those protocols rely on assumptions which are impractical. First, most sequence-based approaches do not propose an actual rendezvous protocol, assuming instead that the rendezvous is accomplished when two nodes 'hop on a common available channel in the same time slot' [23]. Obviously, provisions must be made to ensure that one of the nodes receives while the other transmits, otherwise a rendezvous can't be accomplished.

Second, most sequence-based approaches rely on synchronization of nodes' clocks and, by extension, their sequences [39]. Making allowances for clock phase shift and drift over time (skew) leads to an extension of time to rendezvous, usually by a constant factor. Furthermore, sequence-based approaches typically require that the nodes attempting to connect follow predefined roles, e.g., one as the so-called initiator, the other as the follower. All of these features requires a central authority which means that these approaches are not truly blind.

Most importantly, most sequence-based rendezvous approaches simply ignore the presence of primary users and the impact of their activity - which is the basic tenet of cognitive communications. The impact of primary user activity is considered in qualitative terms in [12], [36], and in quantitative terms - but to a limited extent only - in [5].

In addition, most papers focus on the scenario in which two or more nodes just find each other and establish communication, instead of the scenario in which a node finds and joins an operational piconet. This approach is similar to that one adopted in Bluetooth [16], where the discovery procedure (as rendezvous is called in Bluetooth) is mutually exclusive with normal operation of the piconet. Yet separation of rendezvous and normal operation is impractical in many scenarios such as emergency network operation, disaster management, military communications, and the like. In all these situations, the piconet must operate in an uninterrupted fashion and still allow new nodes to join when they appear in the vicinity. Attempts towards a piconet-oriented rendezvous protocol, e.g., the network setup protocol from [4], still depend on a Cognitive Base Station (effectively, a central controller) and do not entirely address the problem of avoiding interference from primary users. Therefore, a different approach to rendezvous and its integration with the MAC protocol are needed.

\section{Probabilistic RendezVous in trans- MISSION TAX-BASED MAC PROTOCOL}

Recently, a MAC protocol has been described [29], [34] that may be easily extended with a probabilistic rendezvous mechanism. In this protocol, nodes are organized in piconets managed by a coordinator node, similar to in Bluetooth [16]; any node with sufficient computational capability may take up this role. Time is slotted into basic time units and organized in superframes, assumed to contain a certain number of time units. Most of the time in the superframe is reserved for data exchange, but portions are reserved for administrative purposes such as reporting of sensing results, join/leave and bandwidth reservation requests, beacon and trailer frames. Successive 


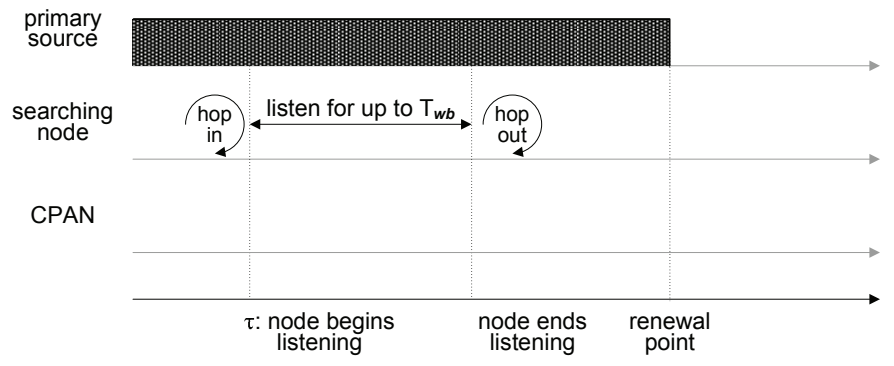

(a) Node is waiting on a busy channel.

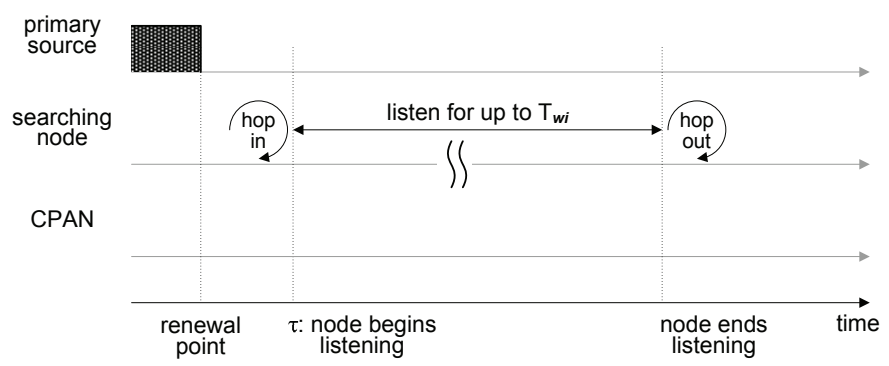

(b) Node is waiting when the piconet is not present on the channel.

Fig. 1. Rendezvous: basic activities of a node that wants to find a piconet.

superframes are separated by a guard interval during which all nodes hop to the next channel. More details about the operation of the protocol are given in Section A of the Online Supplement.

In this protocol, the rendezvous mechanism operates as follows. We assume that all nodes are aware of the MAC protocol and the set of $N$ channels in the working band. (Note that this is not a serious restriction since the nodes would have to use that protocol to communicate on these channels anyway.) Initially, a cognitive node may spend some time hopping through the channels in order to find whether there is an operational piconet in the vicinity. If a piconet can't be found, the node will initiate a new one by beginning to act as a coordinator - i.e., it will begin sending beacon and trailer frames with appropriate administrative information, and hop through available channels in a pseudo-random manner. The required spectrum sensing will initially be performed by the coordinator during the data exchange subframe (see Section A of the Online Supplement for details). As other cognitive nodes join the piconet using the rendezvous protocol, they will begin to exchange data and subsequently perform sensing, and thus gradually take over the sensing function.

A newly arrived node that wants to find an operational piconet must also hop randomly through the channels, as random hopping was shown to be the most efficient approach to rendezvous [3].

- The node may hop to a channel which is busy, i.e., there is a primary user active on that channel, as shown in Fig. 1(a). In this case, the node stays until it is certain about the presence of primary user. Time-wise, the node stays on a busy channel for only a short time period, $T_{w b}$, hereafter referred to as the busy timeout.

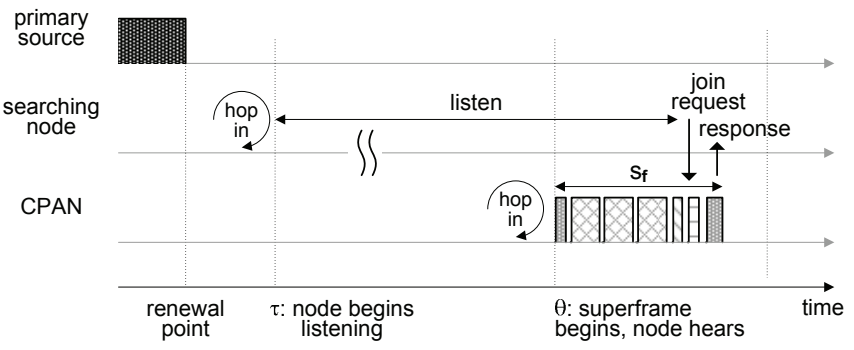

(a) Node hops in before the piconet.

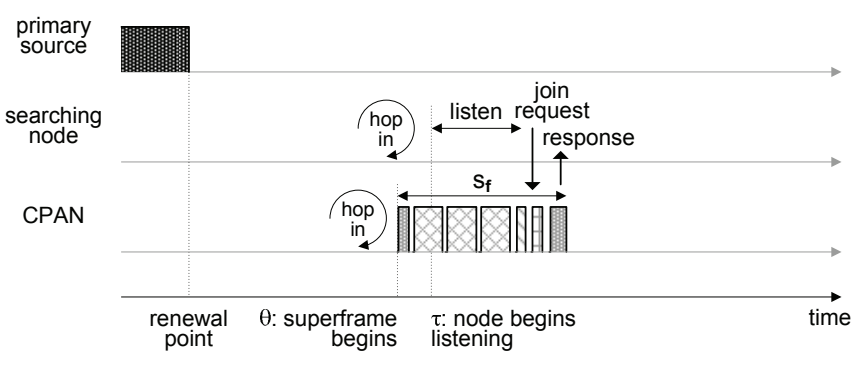

(b) Node hops in after the piconet, but before join/reservation period.

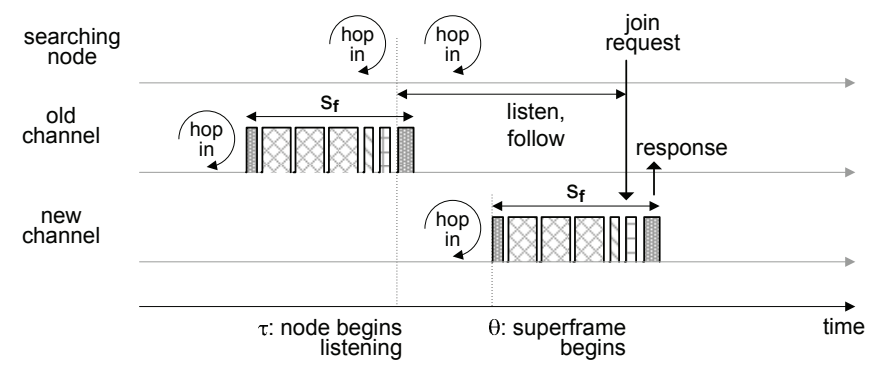

(c) Node hops in just in time to hear the trailer, follows the piconet to the next superframe to join.

\section{Fig. 2. Scenarios for a successful rendezvous.}

- The node may also hop to an idle channel, in which case it will stay there for a longer time, hoping that the piconet will eventually hop in to the same channel so that the communication between the two can be established. The maximum residence interval in this case is referred to as the idle timeout, $T_{w i}$, as shown in Fig. 1(b).

The choice of values for the timeouts $T_{w i}$ and $T_{w b}$ will be discussed in Section 5.1.

Rendezvous succeeds in the following scenarios:

1) The node hops in to the channel and begins to wait for piconet transmissions. Later on, the piconet hops in and begins a superframe. The node recognizes the presence of the piconet and sends a request to join the piconet; the coordinator grants the request and announces the presence of the new node in the trailer. This scenario is shown in Fig. 2(a). If the node has heard a transmission from a piconet, it may prolong its stay beyond the time $T_{w i}$ in order to send its join request and hear the admission decision in the trailer.

2) The node may hop to the channel on which the superframe has already started. Rendezvous may be achieved as long as the node can send in the join request in the reservation subframe, as shown in Fig. 2(b). 


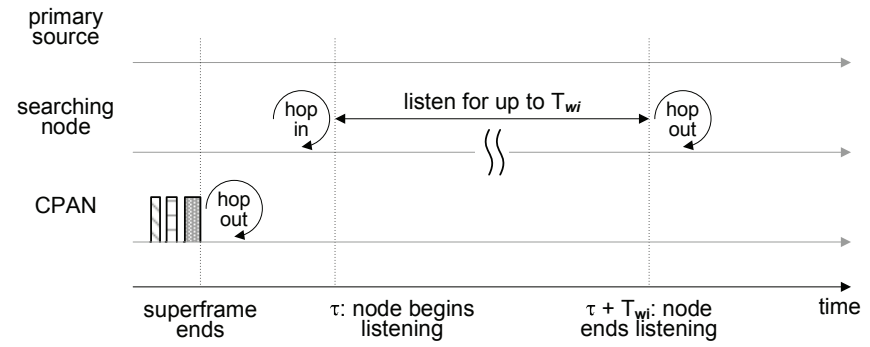

(a) Node hops to the channel after the piconet has already completed superframe on the channel.

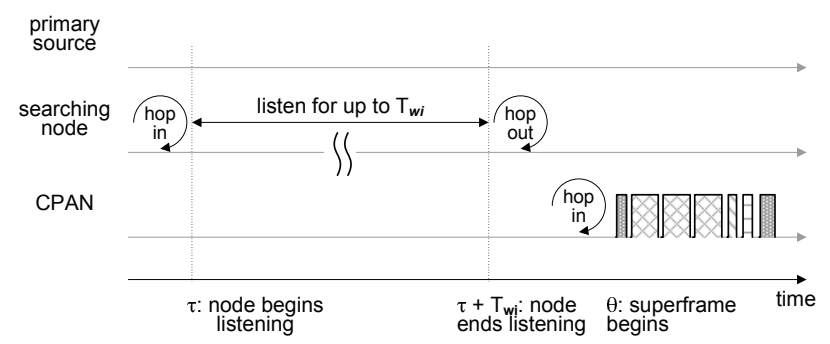

(b) Node has completed waiting before the piconet hopped in to the channel.

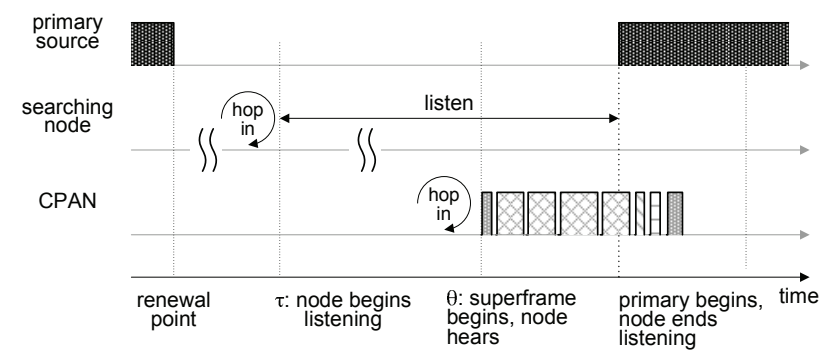

(c) Rendezvous is destroyed by the onset of primary source activity.

Fig. 3. Rendezvous may not occur even when the node and piconet access the same channel.

3) Rendezvous can be achieved as long as the node hops to the channel just in time to hear the trailer, as shown in Fig. 2(c). While the join request can't be sent in that superframe, the node will be able to get the information about the next-hop channel from the trailer so it can follow the piconet to that channel and send its join request in the next superframe.

Rendezvous fails in the following scenarios:

1) The node visits an idle channel but the piconet superframe was completed before the node has arrived, as shown in Fig. 3(a).

2) The node visits an idle channel and complete its idle timeout before the arrival of the piconet, as shown in Fig. 3(b).

3) Finally, the node visits an idle channel and attempts to make a rendezvous with the piconet, but the communication between the two (as well as the communication within the piconet itself) is effectively destroyed by the onset of primary source activity, as shown in Fig. 3(c).

The complete analytical model of the probabilistic rendezvous process is given in Section B of the Online Supplement. This model allows us to calculate the mean value and

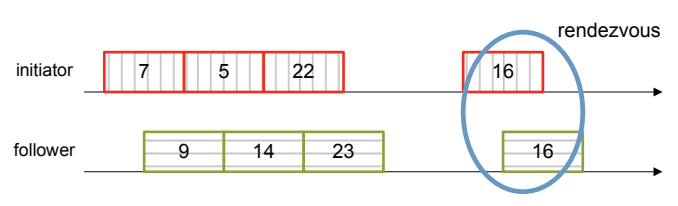

(a) Rendezvous occurs when the nodes hop to the same channel.

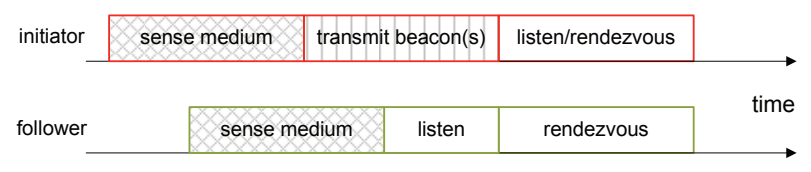

(b) Rendezvous requires that the listen/rendezvous sub-slot of one node overlaps with the beacon transmit slot of the other (after [36]).

Fig. 4. Rendezvous requires synchronization.

variance of the maximum TTR; in fact, the entire probability distribution function (PDF) can be calculated using inverse Laplace transform [17].

\section{Sequence-based Rendezvous}

Most sequence-based rendezvous mechanisms assume that two nodes hop through a common set of $N$ channels according to a predefined sequence. The first node to begin is called the initiator, the other one the follower. (Following [5], [6], [21], [36], we assume that the nodes are aware of their roles.) However, the nodes need not begin the sequence at the same time, nor do they need to follow the same sequence. The sequence of the follower node will lag the one of the initiator node by a time between 0 and $s_{l}-1$ rendezvous slots, where $s_{l}$ denotes the length of the sequence expressed in rendezvous slots. (A rendezvous slot or r-slot is the time interval during which a node stays on a single channel in the hopping sequence.) When the nodes hop to the same channel at the same time, a rendezvous occurs, as shown in Fig. 4(a). The synchronization of channel hopping sequences is, thus, the first level of synchronization; this is the problem that all sequence-based algorithms focus on.

The second, lower level of synchronization must be achieved within the rendezvous time slot itself. Namely, the protocol must ensure that the follower node will be listening to the channel at the time when the initiator is transmitting its beacon on that same channel. Upon hearing the beacon, the follower initiates negotiation that should ultimately result in the establishment of the link between the two nodes. An example of rendezvous slot structure that takes clock skew into account is shown in Fig. 4(b) (adapted from [36]). From this diagram, we may infer that the rendezvous slot for the initiator node should consist of medium sensing, beacon transmit, and listen/rendezvous sub-slots, while the corresponding slot for the follower node should consist of medium sensing, listen and rendezvous sub-slots, the last two of which could be lumped into a single listen/rendezvous slot. The media sensing slot is needed so that the node can establish that the channel is idle and rendezvous can occur; otherwise the node will remove that channel from its sequence, recalculate the sequence and restart it. Note that initiator and follower will update their sequences 


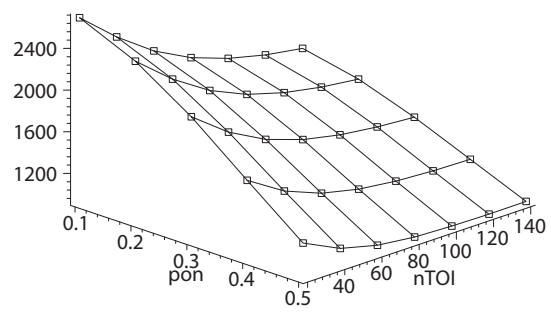

(a) Mean TTR.

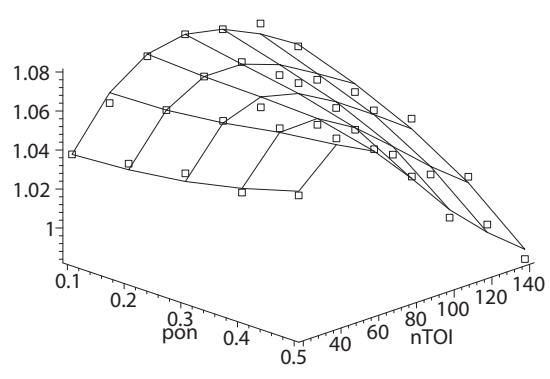

(b) Coefficient of variation of TTR.

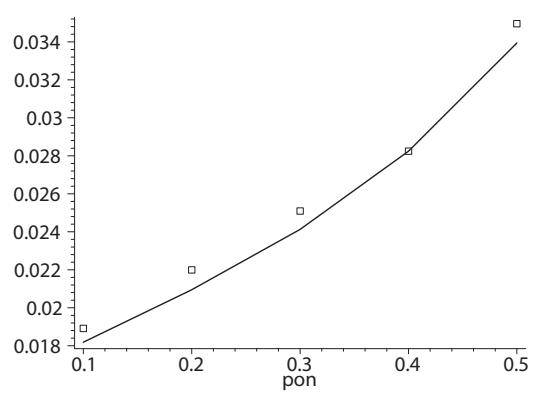

(c) Collision probability.

Fig. 5. Performance of probabilistic rendezvous in an emergent piconet with $N=25$ channels and cycle time of primary sources of $\overline{T_{c y c}}=3000$. Lines: analytical results; boxes: simulation results.

at different times, as they will not visit busy channels at the same time. Still, some sequences are guaranteed to lead to a rendezvous as long as there is at least one idle channel [12], [36].

The duration of sub-slots relative to the rendezvous slot may be determined by noting that the listen/rendezvous subslot of the follower must overlap with the beacon transmit sub-slot of the initiator. The overlap is ensured if the follower node listen/rendezvous sub-slot is not shorter than the sum of initiator node medium sense and listen/rendezvous subslots; in this case, the skew between the node clocks (or, more precisely, between their hopping sequences) will not affect the rendezvous procedure. Shorter durations of the follower node listen/rendezvous sub-slot will result in a dead time which may preclude the rendezvous even when the nodes hop to the same channel at nearly the same time.

Based on these considerations, we may assume that the medium sense sub-slot, transmit beacon, and listen/rendezvous slots of the initiator last about the same time, say $\delta$ basic time units, while the sense medium and listen/rendezvous slots of the follower node last for $\delta$ and $2 \delta$ basic units, respectively. Then, the total duration of the r-slot is $s_{r}=3 \delta$ basic time units. In absolute terms, the duration $\delta$ of the initiator node listen/rendezvous sub-slot must be long enough to allow for successful establishment of the link between the two nodes.

However, under random primary user activity, a pending rendezvous may be destroyed by primary user activity, on account of which TTR becomes a random variable. To characterize its probability distribution, we have modeled the duration of rendezvous sequence broken by random primary user activity. The details of the probabilistic model are given in Section C of the Online Supplement; ultimately, our model allows us to calculate the mean value and variance of the maximum TTR.

\section{Rendezvous Protocol performance}

To evaluate the performance of rendezvous algorithms, we have developed analytical models for both probabilistic rendezvous mechanism in a piconet operating under transmission tax-based MAC protocol and the sequence-based rendezvous mechanism from the previous Section. The development of the models is described in detail in the Online Supplement.
We have then solved the models using Maple 13 software package by Maplesoft, Inc. [27]. To validate the analytical results, we have built discrete event simulators of the two mechanisms using object-oriented Petri Net-based simulation engine Artifex by RSoftDesign, Inc. [35]. All diagrams show both analytical results, shown with lines, as well as those obtained through simulation, shown with boxes.

We assume that channel idle and busy times due to primary user activity are exponentially distributed with average values $\overline{T_{i}}$ and $\overline{T_{a}}$ respectively. Primary user activity factor $p_{o n}=$ $\frac{\overline{T_{a}}}{\overline{T_{a}}+\overline{T_{i}}}$ was varied in the range 0.1 to 0.5 , while the mean cycle time was set to $\overline{T_{c y c}}=\overline{T_{i}}+\overline{T_{a}}=3000$ or 6000 time units.

\subsection{Probabilistic rendezvous in an emergent piconet}

Our first experiment concerns a piconet with the transmission tax-based MAC protocol operating in the 'emergent' mode, i.e., with the coordinator emitting beacon frames and hopping through the channels, but without any data traffic. This scenario corresponds to the one-node-seeks-another-node scenario that is typical for sequence-based rendezvous algorithms.

In the absence of data traffic, the superframe duration was set to $s_{f}=50$ time units, a portion $(\Delta=20)$ of which was set aside for reservation and join requests, beacon, trailer, and guard intervals. The coordinator performs the sensing itself during the data transmission sub-frame.

Sensing of one channel, including the time needed to switch to the channel, was assumed to take $d_{s}=5$ time units. Shorter sensing interval would improve the accuracy of spectrum sensing but not its timeliness [28]; as the result, there would still be non-zero probability of a collision of either type, and our analysis would still hold.

As both channel cycle time and superframe duration are expressed in relative units, our analysis is applicable in a wide range of real frequency bands.

The parameters of the rendezvous protocol were set as follows: the busy timeout was fixed at $T_{w b}=10$ time units [32] while the idle timeout was set to the product of the number of primary channels and normalized timeout parameter $n T O I$, i.e., $T_{w i}=n T O I \cdot N$. To determine the optimal choice of $n T O I$, we have considered the piconet operating in a band 


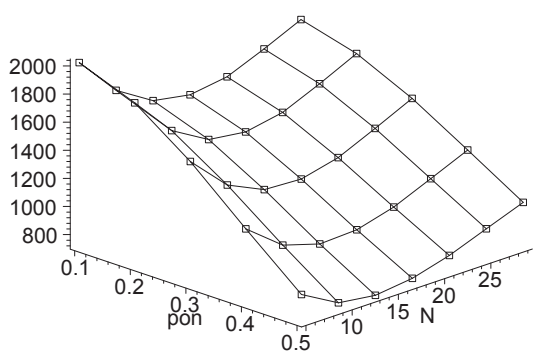

(a) Mean TTR.

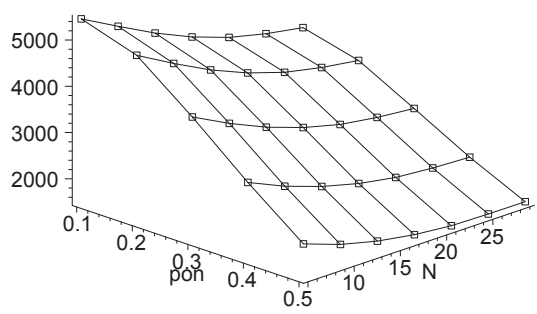

(d) Mean TTR.

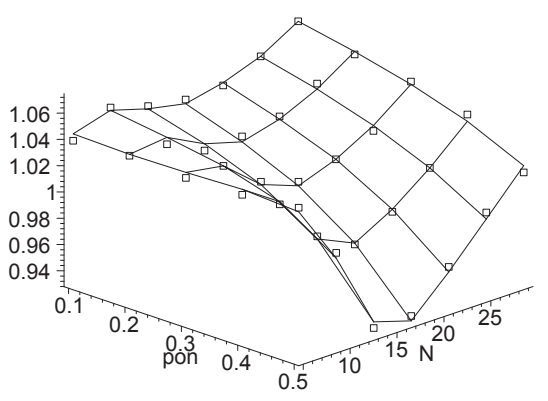

(b) Coefficient of variation of TTR.

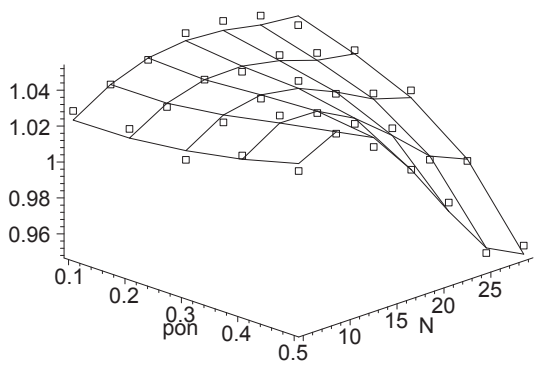

(e) Coefficient of variation of TTR.

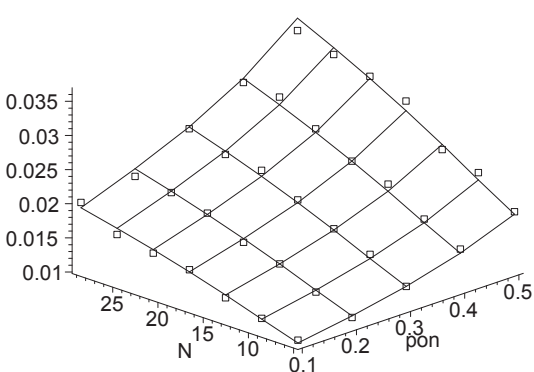

(c) Collision probability.

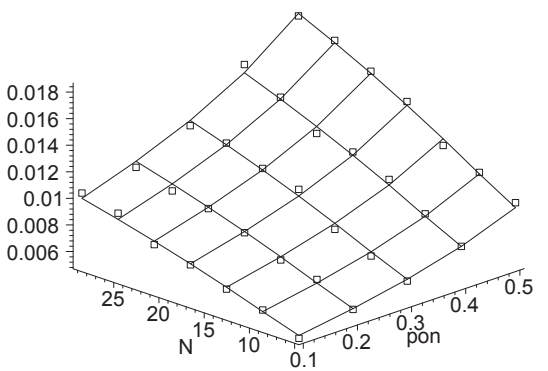

(f) Collision probability.

Fig. 6. Performance of probabilistic rendezvous in an emergent piconet with normalized idle timeout $n T O I=140$ and cycle time of primary sources of $\overline{T_{c y c}}=3000$ (top row) and $\overline{T_{c y c}}=6000$ (bottom row). Lines: analytical results; boxes: simulation results.

with $N=25$ channels and mean primary user cycle time $\overline{T_{c y c}}=3000$ time units, and varies the primary user duty cycle and normalized idle timeout. The diagrams in Fig. 5 shows the mean value and coefficient of variation of TTR, respectively, and probability of collision $P_{c o l}$.

As can be seen, mean TTR decreases with an increase in primary user duty cycle. This is not unexpected since larger values of $p_{\text {on }}$ correspond to shorter mean idle time $\overline{T_{i}}=\left(1-p_{o n}\right) T_{c y c}$, which, in turn, increases the probability of overlap between piconet and node residence times conditioned on their meeting at an idle channel. Collision probability also increases, but its degrading effect is more than compensated for by the increase in $P_{o v}$. At the same time, mean TTR decreases when normalized timeout $n T O I$ increases; the effect is more pronounced at larger channel idle times. Coefficient of variation of rendezvous time is in the range of 1 to 1.08 which indicates that the distribution of rendezvous time is exponential or mildly hyperexponential.

On account of these results, we have chosen to use the value of $n T O I=140$ for the normalized idle timeout of the probabilistic rendezvous algorithm.

To evaluate the impact of primary user cycle time, we have then solved the model for $\overline{T_{c y c}}=3000$ and 6000 time units under variable number of channels and primary user duty cycle. The corresponding performance descriptors are shown in Fig. 6. For smaller cycle time $\overline{T_{c y c}}=3000$ (diagrams in the top row), TTR has a minimum around 13 channels, while for $\overline{T_{c y c}}=6000$ (bottom row) rendezvous time monotonically decreases when the number of channels increases. Minimal values of mean TTR are typically accompanied by subexponential values of the coefficient of variation of TTR.
The probability of collisions between piconet and primary source increases when the activity factor of the primary source is increasing, as might be expected. The probability of collisions also increases when the number of channels increases, on account of an increase in the probability that a channel is incorrectly considered to be idle in the channel map caused by sensing errors [28], [31]. Namely, for a constant number of piconet nodes randomly sensing $N$ channels, the period of sensing of a given target channel (which is a random variable) increases with the number of channels, which in turn leads to an increase of the probability that a change in channel status will not be immediately noticed.

However, increased collision probability is balanced by the increase in probability of time overlap between the node and piconet which increases in the same range since the idle timeout value $T_{w i}=n T O I \cdot N$ increases linearly with the number of channels. Probability of overlap also increases when idle time decreases (i.e., with the increase of activity factor) since there is less time for overlap.

Collision probability decreases with the increase of cycle time $\overline{T_{c y c}}$, as expected, while the coefficient of variation is slightly lower when the cycle time of primary sources increases since the variation of unsuccessful waiting time decreases in size.

We note that the match between analytical and simulation results is quite good, which confirms the validity of the developed analytical model.

\subsection{Sequence-based rendezvous}

To evaluate the performance of sequence-based approach, we have used the orthogonal sequence algorithm from [12], [36] 


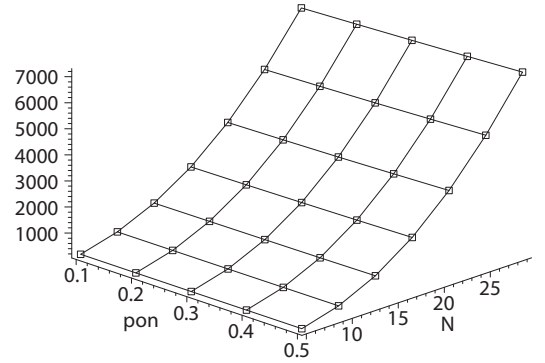

(a) Mean TTR.

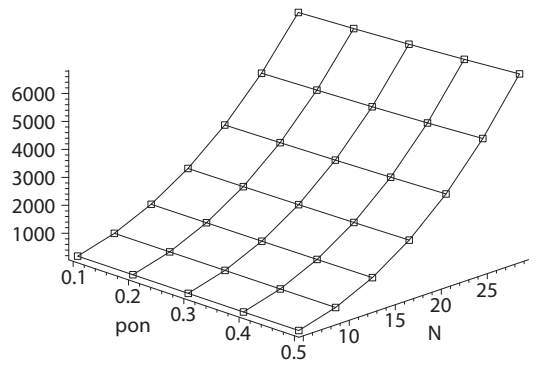

(d) Mean TTR.

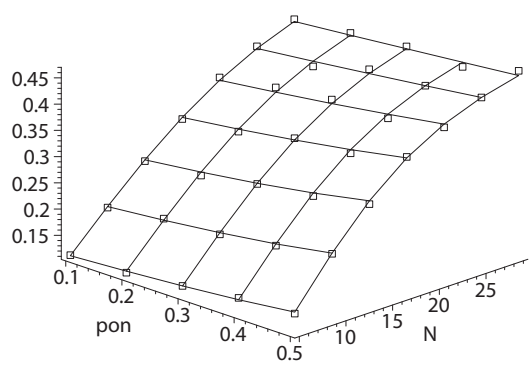

(b) Coefficient of variation of TTR.

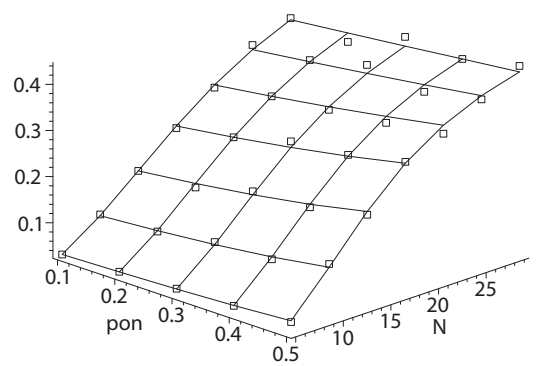

(c) Probability that rendezvous will be destroyed.

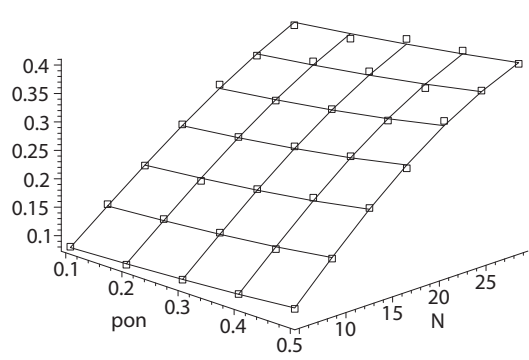

(e) Coefficient of variation of TTR.

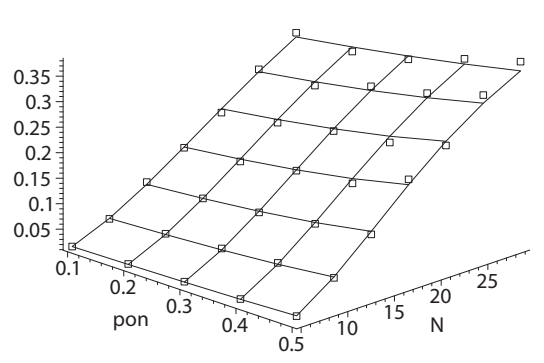

(f) Probability that rendezvous will be destroyed.

Fig. 7. Performance of sequence-based rendezvous as functions of number of channels and activity factor of the channel (top row: at $\overline{T_{c y c}}=3000$ and $s s=3 \delta=6$; bottom row: $\overline{T_{c y c}}=6000$ and $s s=3 \delta=6$ ). Lines: analytical results; boxes: simulation results.

in which the sequence is obtained by interspersing a random permutation of the channel set with individual channels from that permutation. For a network with $N$ channels, this sequence contains $s_{l}=N(N+1)$ rendezvous slots, which is also the upper bound for the rendezvous time. We stress that this algorithm is used only as a representative for the general class of sequence-based approaches - other sequence-based algorithms will be affected by primary user activity in a similar manner.

In the evaluation of the sequence-based algorithm, the duration of rendezvous sub-slot $\delta$ must be sufficient to detect channel activity or submit a rendezvous request. Therefore, we have set $\delta=2$ time units, while the duration of r-slot was $s_{r}=3 \delta=6$ time units. We did not address the problem of clock skew between initiator and follower since it can be overcome by a suitable phase shifting algorithm. Also, we did consider potential collisions with primary user activity on the rendezvous channel only.

The main measure of the impact of primary user activity on the performance of sequence-based rendezvous algorithms is the probability that the sequence-based rendezvous will be destroyed by onset of activity of primary user between the moment when the initiator starts the sequence and the moment where rendezvous actually occurs. We have also calculated the expected value (i.e., mean) and variance of TTR when primary users are active.

Fig. 7 shows the performance descriptors for primary user cycle time of $T_{c y c}=3000$ and 6000 in the top and bottom row of diagrams, respectively. Probability that a rendezvous will be destroyed increases significantly with the number of channels, reaching as much as 0.4 at $N=29$ channels in both diagrams. While it may seem counterintuitive that more channels should result in greater chance that a rendezvous will be destroyed, one should keep in mind that the length of the orthogonal sequence increases as the square of the number of channels, thus more channels lead to much longer sequences and, consequently, much higher probability of collision.

The same general trend may be observed for mean value and coefficient of variation of TTR. Note that the mean value is noticeably higher, e.g., over 900 time units, than the theoretical bound of $3 s_{l} \delta=6 N(N+1)=660$ time units at $N=10$ channels, while the coefficient of variation, albeit smaller than one, increases rapidly with the number of channels.

The increase is less dependent on the increase of primary user duty cycle, which corresponds to a reduction in channel idle time. Collision probability drops with the increase of cycle time of primary source. However, doubling the cycle time reduces the collision probability by only about 10 to $12 \%$, with the corresponding decrease in mean and coefficient of variation of TTR, which may be explained by the exponential distribution of channel idle time.

Again, the simulation results closely match the analytical ones.

\subsection{Comparison of algorithms}

As can be seen, probabilistic and sequence-based rendezvous algorithms have quite different behavior with respect to several parameters. From the diagrams in Figs. 6 and 7, mean TTR is smaller for the probabilistic algorithm when the number of channels exceeds 15 , regardless of the primary user activity 
factor. As the number of channels increases, the difference grows sharply; beyond 20 channels or so, the mean TTR of the sequence-based algorithm is more than twice the value obtained for the probabilistic algorithm. The main reason for this behavior is that sequence length in sequence-based algorithm grows quadratically with the number of channels, whereas the superframe duration is constant in the probabilistic algorithm while the node waiting time on an idle channel is a linear function of the number of channels.

Another source of discrepancy is the behavior in case of a destroyed rendezvous: in case of the sequence-based algorithm, both initiator and follower nodes repeat their respective sequences until they eventually meet. As the relative lag between the two sequences is fixed, the new opportunity for rendezvous will occur exactly $s_{l}$ slots after the destroyed one - but that opportunity (and other subsequent ones) may also be destroyed through collision with primary user transmission. As the result, the mean TTR (which becomes a random variable) increases substantially.

In the probabilistic algorithm, on the other hand, the node that collides will simply move on to another channel, as does the piconet upon encountering a collision. Since piconet hops rather quickly through the channels due to short superframe size, and node waiting time on the idle channel $T_{w i}=n T O I \cdot N$ increases only linearly with the number of channels, probability that the node will meet the piconet actually increases with the number of channels, which in turn reduces mean TTR.

Quite the opposite behavior may be observed for the coefficient of variation of TTR, which is lower for the sequencebased algorithm. The value of the coefficient of variation of TTR for the probabilistic algorithm is around 1, which indicates that the distribution of rendezvous time is approximately exponential. Again, this might be expected since the TTR for the probabilistic algorithm depends on many heterogenous events (as described in Section B of the Online Supplement), while the TTR for the sequence-based algorithm depends mainly on the primary user activity and the random lag between the beginning of initiator and follower sequences.

Similar behavior may be observed at $\overline{T_{c y c}}=6000$. Again, the probabilistic algorithm performs worse than the sequencebased one when the number of channels is small (e.g., below 15) since the node waiting time is relatively short compared to the channel idle time. However for larger number of channels, the probabilistic algorithm outperforms its sequence-based counterpart algorithm in terms of mean TTR. As before, the coefficient of variation is close to 1 for the probabilistic algorithm, while it does not exceed 0.4 for sequence-based algorithm.

We may conclude that for small channel idle times and large number of channels, probabilistic algorithm is the clear winner, and it is still a better choice (although by a smaller margin) for larger channel idle times. Sequence-based algorithm is preferable only when channel idle times are very long and the number of channels is small.

While our analysis has focused on one specific sequencebased algorithm, other similar algorithms will be affected by random primary user activity in the same manner. Namely, their respective TTR's will become random variables without a finite upper bound, and our analysis and our conclusions apply, in qualitative terms at least, for these algorithms as well. However, the operational details of other algorithms as well as their upper bound for TTR will, of course, differ from those of the orthogonal sequence algorithm.

\subsection{Probabilistic rendezvous in a fully operational piconet}

Our final experiment deals with the scenario in which a node attempts to find and rendezvous with a fully operational piconet that uses the transmission tax-based MAC protocol. This scenario has no counterpart in the sequence-based approach. The piconet has $M=16$ nodes, each having a buffer of size $K=10$ packets, while the superframe duration was set to $s_{f}=100$ time units. As before, $\Delta=20$ time units were set aside for administrative overhead. Packet arrival process was set to Poisson with arrival rate of $\lambda=0.002$ packets per time unit per node, while packet duration was uniformly distributed between 8 and 12 time units with an average value of $k_{d}=10$. Duration of the acknowledgment packet was set to one time unit. Packet destinations were uniformly distributed over all piconet nodes. Maximum number of packets from a single node that can be serviced in one superframe is $\mu=3$. Transmission tax was set to $k_{p}=4$ superframes per transmission, regardless of the number of packets sent. Analytical results have been validated through discrete event simulation, as outlined above.

Basic performance descriptors for this setup are shown in Fig. 8. As can be seen, mean TTR and its coefficient of variation increase only slightly in comparison with the emergent piconet, mainly on account of higher collision probability caused by the longer superframe. We note that collision probability decreases with increase of channel cycle time $\overline{T_{\text {cyc }}}$; however, since unsuccessful waiting time increases, mean TTR will increase as well, while the coefficient of variation exhibits a slight decrease. As before, higher primary user duty cycle leads to shorter channel idle times, which in turn lead to a decrease in mean TTR. As might be expected, the coefficient of variation slightly increases with the number of channels and decreases when activity factor is growing, while the collision probability increases with increasing activity factor and number of channels. As the coefficient of variation of TTR takes values in the range 1.0 to 1.14 , the distribution of TTR can be considered mildly hyperexponential. Also, the agreement between analytical and simulation results is very good.

Collision probability decreases with increase of channel cycle time $\overline{T_{c y c}}$, which is to be expected. As unsuccessful waiting time increases when cycle time of primary source grows, mean TTR will also increase. However, coefficient of variation is slightly smaller when the channel cycle time increases since variation of unsuccessful waiting time is becoming smaller.

On the whole, performance of the probabilistic rendezvous is only slightly worse in the fully operational piconet scenario vs. that in the emergent piconet scenario. This corroborates our thesis that probabilistic rendezvous, while inferior to the 


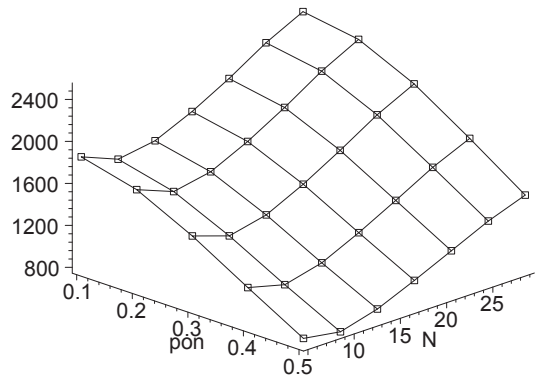

(a) Mean TTR.

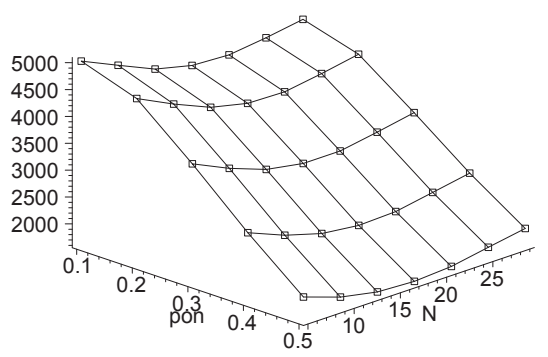

(d) Mean TTR.

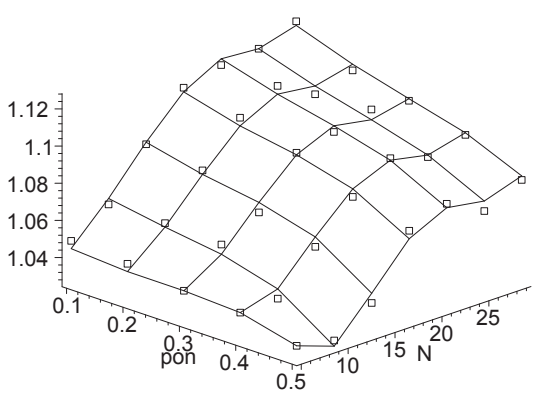

(b) Coefficient of variation of TTR.

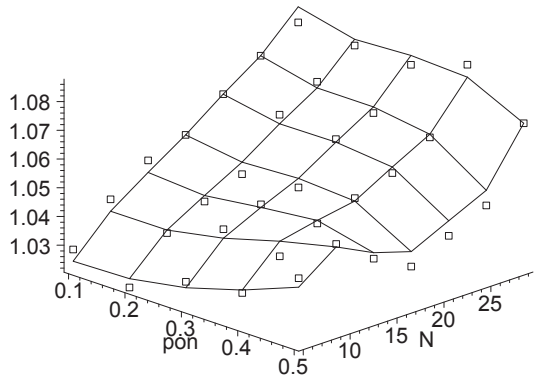

(e) Coefficient of variation of TTR.

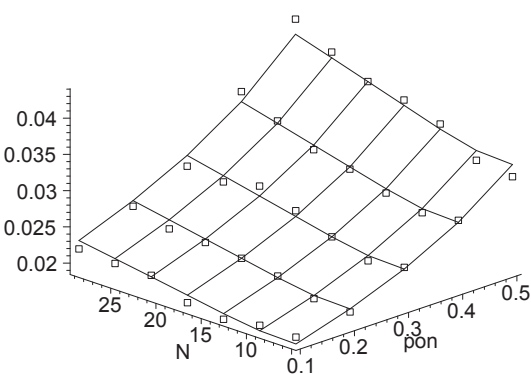

(c) Collision probability between piconet and primary source.

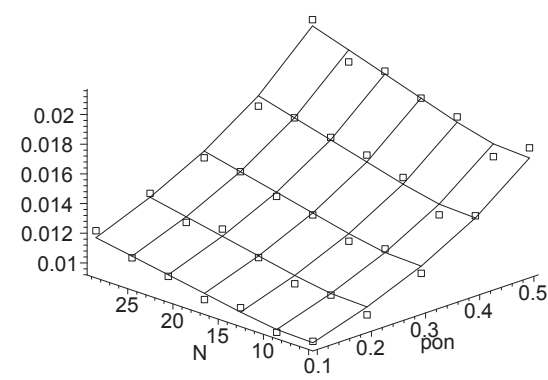

(f) Collision probability between piconet and primary source.

Fig. 8. Performance of probabilistic rendezvous in a fully operational piconet with normalized idle timeout $n T O I=140$ and cycle time of primary sources of $\overline{T_{c y c}}=3000$ (top row) and $\overline{T_{c y c}}=6000$ (bottom row). Lines: analytical results; boxes: simulation results.

sequence-based rendezvous in the ideal case, is much more resilient under realistic conditions that include random activity of primary users.

It is worth noting that the duration of the superframe $\left(s_{f}=50\right.$ time units) is an order of magnitude smaller than the mean channel idle time, $\overline{T_{i}}=p_{o f f} \cdot T_{c y c}$. Reducing mean channel idle time increases mean TTR, mostly through an increase in the probability of type 2 collisions, $P_{\text {col }}$, which is calculated in the Online Supplement. In case of a collision, an emergent piconet will simply move to the next channel after the busy timeout, $T_{w b}$, while an operational piconet will lose the ongoing superframe and will have to undertake a new rendezvous procedure. The need for a full rendezvous can be alleviated by a so-called recovery procedure which strives to resume normal operation much more quickly, however this is beyond the scope of the present paper; more details can be found in [30].

\section{Conclusion}

In this paper we have evaluated the performance of probabilistic rendezvous algorithm in the context of a simple transmission tax-based MAC protocol for channel hopping cognitive personal area networks, and compared it to the performance of a representative sequence-based rendezvous algorithm. The probabilistic algorithm was found to outperform the sequence-based one in a wide range of parameter values. Moreover, the probabilistic algorithm allows a node to discover an operational piconet which does not even need to suspend its operation during the rendezvous procedure, which can offer distinct advantages in many practical scenarios. Mean TTR is shown to depend mostly on the number of channels and primary user activity factor, and to a somewhat lesser extent to the primary user cycle time. We stress that time to rendezvous in sequence-based algorithms has a finite upper bound in the absence of primary user activity, but becomes a random variable without a finite upper bound under random activity of primary users.

Our future work will focus on designing recovery algorithms that will allow a transmission tax-based CH-CPAN piconet to resume normal operation upon collision with a transmission from a primary user. We also plan to work on estimation of primary user activity patterns, esp. in cases where primary user activity does not follow a memoryless probability distribution.

\section{REFERENCES}

[1] I. F. Akyildiz, W.-Y. Lee, M. C. Vuran, and S. Mohanty. NeXt generation/dynamic spectrum access/cognitive radio wireless networks: A survey. Computer Networks, 50:2127-2159, 2006.

[2] A. Alshamrani, X. S. Shen, and L.-L. Xie. A cooperative MAC with efficient spectrum sensing algorithm for distributed opportunistic spectrum networks. Journal of Communications, 4(10):728-740, 2009.

[3] E. Anderson and R. Weber. The rendezvous problem on discrete locations. J. Applied Probability and Statistics, 28:839-851, 1990.

[4] C. J. L. Arachchige, S. Venkatesan, and N. Mittal. An asynchronous neighbor discovery algorithm for cognitive radio networks. In 3rd IEEE Int'l Symp. on New Frontiers in Dynamic Spectrum Access Networks DySPAN 2008, Chicago, IL, Oct. 2008.

[5] K. Bian and J.-M. Park. Maximizing rendezvous diversity in rendezvous protocols for decentralized cognitive radio networks. IEEE Transactions on Mobile Computing, 12(7):1294-1307, 2012. 
[6] K. Bian, J.-M. Park, and R. Chen. Control channel establishment in cognitive radio networks using channel hopping. IEEE J. on Selected Areas in Communications - Wireless Series, 29(4):689-703, 2011.

[7] V. Brik, E. Rozner, S. Banerjee, and P. Bahl. DSAP: a protocol for coordinated spectrum access. In IEEE Int'l Symp. on New Frontiers in Dynamic Spectrum Access Networks DySPAN 2005, pages 611-614, Baltimore, MD, Nov. 2005.

[8] Q. Chen, Y.-C. Liang, M. Motani, and W.-C. Wong. A two-level MAC protocol strategy for opportunistic spectrum access in cognitive radio networks. IEEE Transactions on Vehicular Technology, 60(5):21642180, 2011.

[9] C. Cordeiro, K. Challapali, D. Birru, and N. Sai Shankar. IEEE 802.22: the first worldwide wireless standard based on cognitive radios. In IEEE Int'l Symp. on New Frontiers in Dynamic Spectrum Access Networks DySPAN 2005, pages 328-337, Baltimore, MD, Nov. 2005.

[10] C. Cormio and K. R. Chowdhury. A survey on MAC protocols for cognitive radio networks. Ad Hoc Networks, 7(7):1315-1329, 2009

[11] C. Cormio and K. R. Chowdhury. Common control channel design for cognitive radio wireless ad hoc networks using adaptive frequency hopping. Ad Hoc Networks, 8(4):430-438, 2010.

[12] L. DaSilva and I. Guerreiro. Sequence-based rendezvous for dynamic spectrum access. In 3rd IEEE Symposium on New Frontiers in Dynamic Spectrum Access Networks (DySPAN 2008), pages 1-7, Oct. 2008.

[13] Y. Duan, G. Liu, and Z. Cai. Opportunistic channel-hopping based effective rendezvous establishment in cognitive radio networks. In Wireless Algorithms, Systems, and Applications, pages 324-336, 2012.

[14] R. Gandhi, C.-C. Wang, and Y. C. Hu. Fast rendezvous for multiple clients for cognitive radios using coordinated channel hopping. In 9th Conf. Sensor, Mesh and Ad Hoc Communications and Networks (SECON'2012), pages 434-442, June 2012.

[15] B. Horine and D. Turgut. Link rendezvous protocol for cognitive radio networks. In 2nd IEEE Int'l Symp. on New Frontiers in Dynamic Spectrum Access Networks DySPAN 2007, pages 444-447, Dublin, Ireland, Apr. 2007.

[16] IEEE. Wireless medium access control (MAC) and physical layer (PHY) specifications for wireless personal area networks (WPAN). IEEE Std 802.15.1 (Revision of IEEE Std 802.15.1-2002), IEEE, New York, NY, 2005.

[17] L. J. Kleinrock. Queuing Systems, volume I: Theory. John Wiley and Sons, New York, 1972.

[18] Y. Kondareddy, P. Agrawal, and K. Sivalingam. Cognitive radio network setup without a common control channel. In IEEE Military Communications Conference (MILCOM 2008), pages 1-6, 2008.

[19] L. Lai, H. El Gamal, H. Jiang, and H. Poor. Cognitive medium access: Exploration, exploitation, and competition. IEEE Transactions on Mobile Computing, 10(2):239-253, 2011.

[20] P. K. Lee. Joint frequency hopping and adaptive spectrum exploitation. In IEEE Military Communications Conference MILCOM2001, volume 1, pages 566-570, Washington, DC, Oct. 2001.

[21] Z. Lin, H. Liu, X. Chu, and Y.-W. Leung. Jump-stay based channelhopping algorithm with guaranteed rendezvous for cognitive radio networks. In Proc. INFOCOM, pages 2444-2452, Apr. 2011.

[22] Z. Lin, H. Liu, X. Chu, and Y.-W. Leung. Ring-walk rendezvous algorithms for cognitive radio networks. Ad Hoc and Sensor Wireless Networks, 16(4):243-271, 2012.

[23] H. Liu, Z. Lin, X. Chu, and Y.-W. Leung. Ring-walk based channelhopping algorithms with guaranteed rendezvous for cognitive radio networks. In Proc. 2010 IEEE/ACM Int'l Conference on Green Computing and Communications \& Int'l Conference on Cyber, Physical and Social Computing, GREENCOM-CPSCOM '10, pages 755-760, 2010.

[24] H. Liu, Z. Lin, X. Chu, and Y.-W. Leung. Jump-stay rendezvous algorithm for cognitive radio networks. IEEE Transactions on Parallel and Distributed Systems, 23(10):1867-1881, Oct. 2012.

[25] B. F. Lo, I. F. Akyildiz, and A. M. Al-Dhelaan. Efficient recovery control channel design in cognitive radio ad hoc networks. IEEE Transactions on Vehicular Technology, 59(9):4513-4526, 2010.

[26] L. Ma, X. Han, and C.-C. Shen. Dynamic open spectrum sharing MAC protocol for wireless ad hoc networks. In IEEE Int'l Symp. on New Frontiers in Dynamic Spectrum Access Networks DySPAN 2005, pages 203-213, Baltimore, MD, Nov. 2005.

[27] Maplesoft, Inc. Maple 13. Waterloo, ON, Canada, 2009.

[28] J. Mišić and V. B. Mišić. Performance of cooperative sensing at the MAC level: Error minimization through differential sensing. IEEE Transactions on Vehicular Technology, 58(5):2457-2470, June 2009.

[29] J. Mišić and V. B. Mišić. Simple and efficient MAC for cognitive wireless personal area networks. In Proc. Global Telecommunications Conference GLOBECOM'09, Honolulu, HI, Nov. 2009.

[30] J. Mišić and V. B. Mišić. Recovery using backup channels in channelhopping cognitive networks. In 7th Int. Workshop on Wireless Sensor, Actuator and Robot Networks (WiSARN 2013-Spring), Cambridge, MA, May 2013.

[31] J. Mišić. Cooperative sensing at the MAC level in simple cognitive personal area networks. IEEE J. on Selected Areas in Communications - Wireless Series, 30(9):1711-1718, 2012.

[32] J. Mišić, N. Khan, H. Khojasteh, and V. B. Mišić. CSCD: a simple channel scan protocol to discover and join a cognitive PAN. In IEEE Wireless Communications and Networking Conference (WCNC'2012), Paris, France, Apr. 2012.

[33] J. Mišić, H. Khojasteh, N. Khan, and V. B. Mišić. Towards an efficient rendezvous protocol for a cognitive PAN. In IEEE International Conference on Communications (ICC'2012), Ottawa, ON, June 2012.

[34] V. B. Mišić and J. Mišić. Cognitive MAC protocol with transmission tax: Dynamically adjusting sensing and data performance. In Proc. Global Telecommunications Conference GLOBECOM'10, Miami, FL, Dec. 2010.

[35] RSoft Design. Artifex v.4.4.2. RSoft Design Group, Inc., Ossining, NY, 2003.

[36] N. C. Theis, R. W. Thomas, and L. A. DaSilva. Rendezvous for cognitive radios. IEEE Transactions on Mobile Computing, 10(2):216-227, Feb. 2011.

[37] M. Timmers, S. Pollin, A. Dejonghe, L. Van der Perre, and F. Catthoor. A distributed multichannel MAC protocol for multihop cognitive radio networks. IEEE Transactions on Vehicular Technology, 59(1):446-459, 2010.

[38] D. Yang, J. Shin, and C. Kim. Deterministic rendezvous scheme in multichannel access networks. Electronics Letters, 46(20):1402-1404, 302010 .

[39] J. Zhang and Z. Zhang. Initial link establishment in cognitive radio networks without common control channel. In IEEE Wireless Communications and Networking Conference (WCNC 2011), pages 150-155, 2011.

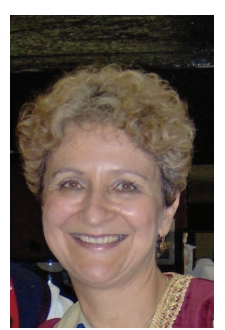

Jelena Mišić (M91, SM08) Professor of Computer Science at Ryerson University in Toronto, Ontario, Canada. She has published over 90 papers in archival journals and more than 120 papers at international conferences in the areas of wireless networks, in particular wireless personal area network and wireless sensor network protocols, performance evaluation, and security. She serves on editorial boards of IEEE Transactions on Vehicular Technology, Computer Networks, Ad hoc Networks, Security and Communication Networks, Ad Hoc \& Sensor Wireless Networks, Int. Journal of Sensor Networks, and Int. Journal of Telemedicine and Applications. She is a Senior Member of IEEE and Member of ACM.

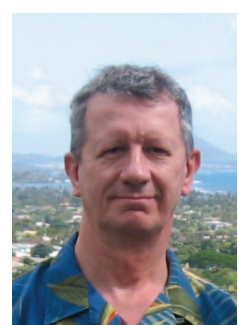

ACM and AIS.
Vojislav B. Mišić is Professor of Computer Science at Ryerson University in Toronto, Ontario, Canada. He received his $\mathrm{PhD}$ in Computer Science from University of Belgrade, Serbia, in 1993. His research interests include performance evaluation of wireless networks and systems and software engineering. He has authored or co-authored six books, 20 book chapters, and over 200 papers in archival journals and at prestigious international conferences. He is a Senior Member of IEEE, and member of 